\title{
MÉTODO QUEChERS PARA DETERMINAÇÃO DE OCRATOXINA A E CITRININA EM ARROZ E FARELO DE ARROZ
}

Helen C. dos S. Hackbart*, Michele Moraes de Souza, Priscila Tessmer Scaglioni, Ednei G. Primel, Jaqueline Garda-Buffon e Eliana Badiale-Furlong

Escola de Química e Alimentos, Universidade Federal do Rio Grande, Alfredo Huck 475, 96200-270 Rio Grande - RS, Brasil

Recebido em 23/11/11; aceito em 18/5/12; publicado na web em 24/8/12

\begin{abstract}
THE QuEChERS METHOD FOR DETERMINATION OF OCHRATOXIN A AND CITRININ IN RICE AND RICE BRAN. The present study was carried out to establish the optimal conditions for performing ochratoxin A (OTA) and citrinin (CIT) extraction using the QuEChERS method in rice. Employing the factorial experimental design, variables that significantly influenced the extraction stages were determined. The following variables were analyzed: addition of water, acidification of acetonitrile with glacial acetic acid, as well as amounts of magnesium sulfate, sodium acetate, sodium citrate and diatomaceous earth. The best combining procedure resulted in a predictive model using more water and less diatomaceous earth. Recoveries of CIT and OTA were 78-105\%.
\end{abstract}

Keywords: ochratoxin A; citrinin; QuEChERS method.

\section{INTRODUÇÃO}

As micotoxinas compreendem uma mistura de compostos resultantes da atividade metabólica de fungos toxigênicos cujos efeitos, além de outros sintomas clássicos de intoxicação, variam entre carcinogenicidade e mutagenicidade. ${ }^{1}$ Estes compostos são resistentes e dificilmente são degradados durante o processamento ou preparo de alimentos e rações. ${ }^{2-4}$

A ocratoxina A e a citrinina são micotoxinas produzidas por fungos dos gêneros Aspergillus e Penicillium, ${ }^{5,6}$ que podem infestar grãos especialmente durante o armazenamento em ambientes com atividade de água superiores a $70 \%$ e temperaturas inferiores a $25^{\circ} \mathrm{C}$. Estas variáveis abióticas são frequentes nas regiões produtoras de cereais, como é o caso do Rio Grande do Sul.

A ocratoxina A (OTA), fórmula molecular $\mathrm{C}_{20} \mathrm{H}_{18} \mathrm{ClNO}_{6}$ e massa molecular de 403,82 $\mathrm{g} / \mathrm{mol}$, é constituída por uma isocumarina, a ocratoxina (OTa), ligada em um grupo amida, L-b fenilalanina. ${ }^{8,9}$ Este composto vem sendo detectado em matérias-primas que foram submetidas a condições que propiciam o desenvolvimento do fungo produtor, ou seja, sob umidade elevada e temperatura de 25 a $30{ }^{\circ} \mathrm{C} \cdot{ }^{10-12}$ Quando o fungo contaminante encontra condições de produzir a toxina, esta permanece estável durante o armazenamento, beneficiamento e preparo para consumo. ${ }^{13}$

A citrinina (CIT) possui fórmula molecular $\mathrm{C}_{13} \mathrm{H}_{14} \mathrm{O}_{5}$, massa molecular de $250,25 \mathrm{~g} / \mathrm{mol}$ e ponto de fusão de $172{ }^{\circ} \mathrm{C} .{ }^{14}$ Este composto tem sido detectado como contaminante em milho, arroz, trigo e outros cereais, ${ }^{15,16}$ em frutas senescentes ${ }^{17}$ e em queijo; esta micotoxina também é estável durante o processamento e preparo de alimentos. ${ }^{18}$

Os métodos utilizando técnicas cromatográficas são os de preferência para a análise e detecção simultânea de multirresíduos, considerando-se a possibilidade de ocorrência simultânea destas micotoxinas. No entanto, para extração simultânea de ambas das matrizes em que ocorrem, vários fatores precisam ser considerados, tais como, granulometria, solvente de extração e eliminação de interferentes. ${ }^{19,20}$ Em consequência disto, as etapas para a determinação propriamente dita são exaustivas, gerando perdas de analito e resíduos para descarte. Encontrar condições adequadas para extração simultânea satisfatória pode requerer muitos experimentos devido

*e-mail: helenhackbart@gmail.com à sutileza do efeito das variáveis abióticas da matriz e do próprio método. Assim, o planejamento experimental e a determinação em CLAE-IEN-EM/EM é uma associação interessante para detectar e ajustar as condições para extração e clarificação. ${ }^{21-23}$

Um método de extração definido como rápido, fácil, barato, eficaz, robusto e seguro, cuja sigla é QuEChERS (Quick, Easy, Cheap, Rugged and Safe), vem sendo adotado para extração de pesticidas de frutas e verduras com o objetivo de superar limitações práticas de outros métodos multirresíduo. ${ }^{24,25}$ Como outros procedimentos de extração ele emprega solventes e agentes clarificantes, como sais e adsorventes, que interagem com a matriz carreando o analito para um solvente e eliminando os interferentes. Sua grande vantagem reside na rapidez e nas quantidades de reagentes necessários. ${ }^{24}$ No entanto, a combinação de agentes extratores, formas de contato entre amostra-extrator e a combinação de agentes de limpeza são pontos críticos para a recuperação de analitos distintos simultaneamente. Alguns autores têm mencionado a possibilidade de aplicá-lo com sucesso na determinação de multimicotoxinas, além do emprego original para pesticidas. ${ }^{25}$

Este trabalho teve como principal objetivo disponibilizar um método acessível para extração simultânea da ocratoxina A e citrinina em arroz e farelo de arroz, empregando a técnica de planejamento experimental para estabelecer condições de extração do método QuEChERS, visando posterior quantificação dessas toxinas em arroz e farelo de arroz.

\section{PARTE EXPERIMENTAL}

\section{Padrões analíticos, reagentes e solventes}

Os padrões analíticos da ocratoxina A e a citrinina foram adquiridas da Sigma Aldrich (São Paulo, Brasil), com pureza $\geq 98 \%$. O metanol (pureza $\geq 99,95 \%$ ) e acetonitrila (pureza ${ }^{3} 99,98 \%$ ) de grau cromatográfico foram adquiridos da Mallinckrodt (Phillipsburg, NJ, USA). O ácido fosfórico (pureza $\geq 85 \%$ ) grau analítico, ácido acético glacial (pureza $\geq 98 \%$ ), hexano (pureza $\geq 96 \%$ ) e benzeno (pureza $\geq 99 \%$ ) foram adquiridos da Merck (Darmstadt, Alemanha). Clorofórmio, cloreto de potássio, cloreto de sódio, sulfato de amônio e sulfato de magnésio foram obtidos da Synth (São Paulo, Brasil). Acetato de sódio e citrato de sódio foram obtidos da Vetec (Rio de 
Janeiro, Brasil) e a terra diatomácea foi obtida da Nuclear (Celite 545, São Paulo, Brasil).

A água ultrapura utilizada no preparo da fase móvel foi obtida por sistema de purificação e filtração Direct-Q UV $3^{\circledR}$ de resistividade $18 \Omega \mathrm{cm}^{-1}$ (Millipore, Bedford, MA, USA).

\section{Preparo das soluções estoque e de trabalho}

Os padrões das micotoxinas foram dissolvidos em benzeno:acetonitrila $(98: 2 \mathrm{v} / \mathrm{v})$, resultando em concentração de $100 \mu \mathrm{g} \mathrm{mL}{ }^{-1}$. As soluções estoque foram diluídas de modo a resultar em soluções de trabalho cujas concentrações $\left(10\right.$ e $50 \mu \mathrm{g} \mathrm{mL}^{-1}$ ) foram determinadas através de espectrofotômetro modelo Cary 100 da Varian (EUA). Os valores da absortividade molar (e) foram 5440 e $5490 \mathrm{~mol}$ $\mathrm{cm}^{-1} \mathrm{e}$ os respectivos comprimentos de onda monitorados foram de 333 e $321 \mathrm{~nm}$ para ocratoxina A e citrinina, respectivamente. ${ }^{26}$ Todas as soluções foram secas e acondicionadas em frascos âmbar a $4-8^{\circ} \mathrm{C}$.

\section{Amostras}

Foram utilizadas 38 amostras de arroz (Oryza sativa, L.) sendo 36 provenientes de grãos cultivados em campos experimentais do Instituto Riograndense do Arroz (IRGA), unidade de Cachoeirinha, Rio Grande do Sul, e outras 2 foram coletadas em silos da região sul do Rio Grande do Sul.

As amostras pertenciam à variedade BR IRGA 417. Após a colheita foram beneficiadas separando-se as frações arroz branco, arroz branco em casca, arroz parboilizado e arroz parboilizado em casca. As amostras provenientes de silos foram beneficiadas separando-se em farelo de arroz e arroz em casca.

\section{CLAE-IEN-EM/EM}

As análises foram realizadas usando um sistema de cromatografia líquida acoplado a um espectrômetro de massas com analisador do tipo triplo quadrupolo (CLAE-EM/EM), cromatógrafo Alliance Separations modelo 2695 (Waters, Milford, MA, USA) equipado com amostrador automático, bomba quaternária, forno para coluna e sistema de desgaseificação; detector MS, Micromass ${ }^{\circledR}$ Quatro Micro $^{\text {TM }}$ API (Waters, Inglaterra) com fonte API. Sistema gerador de nitrogênio Peak Scientifics (Instruments Ltda., Escócia) e coluna

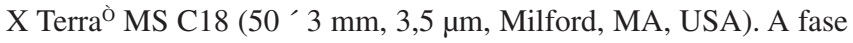
móvel empregada para separação foi constituída por uma mistura de acetonitrila:água ultrapura 60:40 (v/v), eluição no modo isocrático com fluxo de $0,4 \mathrm{~mL} / \mathrm{min}$ e alça de injeção com loop de $10 \mu \mathrm{L}$. Para otimização dos sinais das razões massa/carga foi injetada uma solução de cada micotoxina na concentração de $1 \mu \mathrm{g} / \mathrm{mL}$. O espectrômetro de massas foi operado em modo de ionização por eletronebulização no modo positivo (IEN +) para ocratoxina A e negativo (IEN -) para citrinina utilizando monitoramento de múltiplas reações (MRM). O gás de colisão utilizado foi o argônio com pressão de $3,5^{\prime} 10^{3}$ bar, a temperatura de dessolvatação ótima foi de $350{ }^{\circ} \mathrm{C}$, temperatura da fonte de $120^{\circ} \mathrm{C}$, voltagem do cone de $30 \mathrm{kV}$, voltagem do capilar de $4,0 \mathrm{kV}$ e tempo total de corrida de $7 \mathrm{~min}$. A aquisição dos dados e seu processamento foram realizados através de programa computacional MassLynx 4.0 (Waters, Inglaterra).

Os íons precursores utilizados foram $m / z 404>239\left[\mathrm{M}-\mathrm{C}_{9} \mathrm{H}_{10} \mathrm{NO}_{2}\right]^{+}$ $(+15 \mathrm{eV})$ e $\mathrm{m} / \mathrm{z} 249>205\left[\mathrm{M}-\mathrm{CHO}_{2}\right]^{+}(-12 \mathrm{eV})$ para ocratoxina A e citrinina, respectivamente.

\section{Parâmetros analíticos}

O método utilizando cromatografia foi validado, estabelecendo-se seletividade, linearidade, precisão, limites de detecção e quantificação, parâmetros sugeridos pelo Instituto Nacional de Metrologia, Normalização e Qualidade Industrial ${ }^{27}$ e pela Agência Nacional de Vigilância Sanitária. ${ }^{28}$ A linearidade foi avaliada pelas curvas analíticas com soluções nas concentrações de 0,$005 ; 0,010 ; 0,025 ; 0,050$; 0,$100 ; 0,250 ; 0,500$ e $1,000 \mu \mathrm{g} / \mathrm{mL}$. Cada solução foi injetada três vezes e os dados de regressão linear foram obtidos com auxílio do software do equipamento. A seletividade foi avaliada comparando-se o sinal gerado pela injeção da matriz isenta das micotoxinas e desta adicionada de padrão. ${ }^{29}$

Os limites de detecção (LOD) e de quantificação (LOQ) do instrumento $\left(\mathrm{LOD}_{\mathrm{i}}\right.$ e $L O Q_{\mathrm{i}}$, respectivamente) para cada micotoxina foram estimados considerando-se pelo menos 3 e 10 vezes a razão entre o sinal pela linha de base (ruído), respectivamente. Os limites instrumentais foram obtidos por padronização externa com a preparação de soluções analíticas de diferentes concentrações em acetonitrila. Os valores de $\mathrm{LOD}_{\mathrm{i}}$ e $\mathrm{LOQ}_{\mathrm{i}}$ multiplicados pelos fatores de diluição para o método de extração resultaram nos limites de detecção $\left(\operatorname{LOD}_{\mathrm{m}}\right)$ e quantificação do método $\left(\mathrm{LOQ}_{\mathrm{m}}\right)$ estimado. A precisão instrumental foi avaliada em termos de repetitividade $\left(\mathrm{CV}_{\mathrm{r}}\right)$, a partir de 9 injeções (3 níveis em triplicata) das concentrações dos padrões de 0,$025 ; 0,1$ e $0,5 \mu \mathrm{g} / \mathrm{mL}$.

\section{Adequação do método QuEChERS}

Inicialmente foram realizados 4 ensaios, onde foram testados os efeitos das variáveis formas e tempos de agitação. Para realização dos ensaios foi utilizado o farelo de arroz fortificado com $0,5 \mu \mathrm{g} / \mathrm{mL}$ de ocratoxina A. Os ensaios foram definidos de acordo com a maioria dos métodos multirresíduos de preparo de amostra que empregam agitador de tubos (vórtex), modelo Phenix ò AP 56 (Brasil), mesa agitadora Orbital (shaker) modelo Tecnal ${ }^{\mathrm{O}}$ TE-141 (Brasil) e ultras-

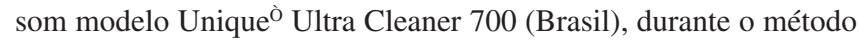
de extração, não ultrapassando um tempo de 30 min.

Escolhida a forma e o tempo de agitação, em seguida, foi realizado um planejamento fatorial, em farelo de arroz fortificado com $0,05 \mu \mathrm{g} /$ $\mathrm{mL}$ de ocratoxina A, onde as variáveis estudadas foram: adição de água, acidificação da acetonitrila com ácido acético glacial, quantidades de: sulfato de magnésio, acetato de sódio, citrato de sódio e terra diatomácea, que foram avaliadas através de planejamento fatorial $2^{4}$ completo com seis pontos centrais, resultando em 22 experimentos.

A área relativa ao sinal da concentração da ocratoxina A foi utilizada para estimar os percentuais de recuperação que constituíram as respostas das variáveis em estudo. A análise estatística dos efeitos foi realizada através do programa Statistica 7.0, utilizando um intervalo de confiança de $90 \%$.

Por fim, na melhor condição do planejamento experimental, foram realizados 6 ensaios, utilizando farelo de arroz fortificado com $0,05 \mu \mathrm{g} / \mathrm{mL}$ de ocratoxina A e citrinina, que tiveram como variáveis: acidificação com ácido acético glacial $(0,1$ e 0,2 mL), quantidade dos sais sulfato de magnésio $(3 ; 1,5 ; 0,85 ; 0,5$ e $0 \mathrm{~g})$, acetato de sódio $(1,7 ; 0,85$ e 0 g) e citrato de sódio $(1 ; 0,5$ e 0 g).

\section{RESULTADOS E DISCUSSÃO}

O sistema CLAE-IEN-EM/EM foi condicionado passando-se a fase móvel acetonitrila:água ultrapura 60:40 (v/v) com eluição no modo isocrático a uma vazão de $0,4 \mathrm{~mL} / \mathrm{min}$ durante alguns minutos. $\mathrm{O}$ método mostrou-se seletivo, pois, a eluição da matriz isenta das micotoxinas em comparação à matriz fortificada indicou que nenhum interferente eluiu no tempo de retenção destas. Na Tabela 1 estão apresentadas as curvas analíticas, linearidade e os limites de detecção e quantificação.

O modelo de regressão linear foi adequado para a determinação das micotoxinas em estudo, pois os coeficientes de correlação (r) 
Tabela 1. Parâmetros analíticos avaliados em CLAE-EM/EM

\begin{tabular}{lcc}
\hline Parâmetros analíticos & Citrinina & Ocratoxina A \\
\hline Linearidade $(\mu \mathrm{g} / \mathrm{mL})$ & $0,025-1$ & $0,005-1$ \\
Coeficiente de correlação & 0,993 & 0,996 \\
Coeficiente de determinação & 0,986 & 0,992 \\
$\mathrm{LOD}_{\mathrm{i}}(\mu \mathrm{g} / \mathrm{mL})$ & 0,01 & 0,001 \\
$\mathrm{LOQ}_{\mathrm{i}}(\mu \mathrm{g} / \mathrm{mL})$ & 0,025 & 0,005 \\
$\mathrm{LOD}_{\mathrm{m}}(\mu \mathrm{g} / \mathrm{kg})$ & 2,8 & 0,3 \\
$\mathrm{LOQ}_{\mathrm{m}}(\mu \mathrm{g} / \mathrm{kg})$ & 7,14 & 1,43 \\
\hline
\end{tabular}

$\mathrm{LOD}_{\mathrm{i}}=$ limite de detecção do CLAE-EM/EM; $\mathrm{LOQ}_{\mathrm{i}}=$ limite de quantificação do CLAE-EM/EM; $\mathrm{LOD}_{\mathrm{m}}=$ Limite de detecção do método; $\mathrm{LOQ}_{\mathrm{m}}=$ limite de quantificação do método.

foram maiores que 0,90 , conforme recomendação do INMETRO. ${ }^{27}$ Os limites de quantificação permitiram determinar concentrações das micotoxinas em níveis inferiores aos estabelecidos pelos órgãos de legislação e fiscalização de diferentes países. Na legislação brasileira, esses níveis são regulados pela Resolução RDC Nº 7 de 2011, sendo para ocratoxina A o limite máximo tolerável (LMT) de $10 \mu \mathrm{g} / \mathrm{kg}$ para cereais e produtos de cereais, incluindo cevada malteada, e $2 \mu \mathrm{g} / \mathrm{kg}$ para alimentos à base de cereais para alimentação infantil (lactentes e crianças de primeira infância).$^{30}$ Alguns autores observaram que a utilização do método QuEChERS foi ideal para obtenção de baixos limites de detecção, além da alternativa de permitir a utilização do extrato bruto para a análise em 11 principais toxinas de Fusarium em amostras de cereais e produtos à base de cereais. ${ }^{31,32}$

$\mathrm{Na}$ União Europeia, o LMT de $3 \mu \mathrm{g} / \mathrm{kg}$ de ocratoxina A foi estabelecido para todos os produtos feitos a partir de cereais não processados para o consumo humano. ${ }^{33}$ Para a citrinina ainda não há limites máximos toleráveis estabelecidos por nenhum órgão de legislação, entre outras razões pela pequena disponibilidade de método analítico e levantamento de ocorrência. Os resultados da precisão instrumental em termos de repetitividade $\left(\mathrm{CV}_{\mathrm{r}} \%\right)$ também atendem as recomendações do limite estabelecido pela ANVISA ${ }^{28}$ de coeficiente de variação (CV) não superiores a $20 \%$ para as duas micotoxinas, variando entre 1,3 e $1,6 \%$ para citrinina e 0,96 e 3,2\% para ocratoxina A.

Em relação à primeira etapa de adequação do método QuEChERS, os resultados apresentados na Tabela 2 mostram que a agitação contínua com a utilização da mesa agitadora orbital (shaker) apresentou a melhor recuperação da ocratoxina A $(95 \%)$ com agitação de 10 min, a 200 rpm, à temperatura ambiente.

As variáveis estudadas no planejamento foram relativas a cada etapa da extração e representavam os pontos críticos para melhorar a extração da ocratoxina A. A adição de água foi estudada para estabelecer a hidratação do tecido celular, facilitando a migração do solvente extrator para o interior da matriz. A diferença na quantidade de sais foi estudada para que ocorresse a diminuição da solubilidade dos compostos na fase aquosa, bem como a quantidade de água na fase orgânica. A acidificação do solvente extrator (acetonitrila) auxilia na estabilidade dos compostos antes da análise cromatográfica e a adição de terra diatomácea foi utilizada para precipitação de coextrativos na fase orgânica.

A Tabela 3 apresenta a matriz do planejamento fatorial estudado, considerando a recuperação da ocratoxina A como resposta das variáveis em estudo.

As melhores recuperações ocorreram nos experimentos 5 e 13, que se diferenciam pela quantidade de água adicionada de 10 e $20 \mathrm{~mL}$, respectivamente.

As análises estatísticas dos efeitos estudados no planejamento $2^{4}$ confirmaram o efeito das associações das variáveis estudadas, demonstrando a importância do estudo detalhado de suas combinações para a confiabilidade do método. $\mathrm{O}$ índice de recuperação indicou a
Tabela 2. Efeito das condições de agitação na recuperação da ocratoxina A

\begin{tabular}{lccc}
\hline Agitação & Tempo (min) & $\begin{array}{c}\text { Coeficiente de } \\
\text { variação }(\%)\end{array}$ & $\begin{array}{c}\text { Recuperação } \\
(\%)\end{array}$ \\
\hline Manual & 0,5 & 8,04 & 43 \\
& 1 & 7,07 & 54 \\
Agitador tubos & 1,5 & 1,08 & 37 \\
& 5 & 1,31 & 36 \\
Agitador orbital & 10 & 5,94 & 40 \\
& 15 & 2,77 & 73 \\
Ultrassom & 5 & 1,71 & 63 \\
& 10 & 1,06 & 95 \\
& 30 & 3,15 & 88 \\
& 5 & 3,21 & 84 \\
& 10 & 0,85 & 90 \\
\hline
\end{tabular}

variável adição de água como significativa, aumentando o efeito da recuperação em 24,6\% ( $\mathrm{p}<0,10)$ quando foi utilizado o maior volume de água adicionada $(20 \mathrm{~mL})$. Estes dados sugerem que a maior eficiência da extração decorreu da hidratação do tecido celular, que deve ter propiciado a migração do solvente extrator para o interior da matriz.

Considerando estas interações de variáveis significativas para a eficiência da extração (maior recuperação), foi possível construir modelo preditivo dentro da faixa avaliada. Estes resultados significativos e preditivos destes parâmetros no percentual de recuperação estão apresentados na Tabela 4 e originaram a Equação 1 e Figura 1.

Recuperação $(\%)=72,8+12,3$ A - 6,1 A.S - 15,3 a.t + 6,5 A.a.t

onde: $\mathrm{A}=$ água; $\mathrm{S}=$ sais; $\mathrm{a}=$ acidificação e $\mathrm{t}=$ terra diatomácea

Os resultados do teste F, no qual o F calculado foi de 9,7, foi 4 vezes maior do que o F tabelado, 2,31 (Tabela 4), permitindo que o modelo expresso na Equação 1 fosse utilizado para gerar a superfície de resposta para a recuperação da ocratoxina A. A Figura 1 mostra que a recuperação foi maior quando se aumentou a quantidade de água adicionada e diminuiu a quantidade de terra diatomácea.

Dos seis ensaios realizados na melhor condição do planejamento, ou seja, o ponto de maior recuperação da ocratoxina A, o ensaio com a maior acidificação $(0,2 \mathrm{~mL})$, maior quantidade de sulfato de magnésio $(1,5 \mathrm{~g})$ e menor quantidade de acetato de sódio $(0,85 \mathrm{~g})$ foi o que apresentou a melhor recuperação da ocratoxina A e citrinina, sendo igual a 105 e $78 \%$, respectivamente.

A acidificação da acetonitrila para extração teve como objetivo minimizar a instabilidade dos compostos estudados em meio básico, ficando demonstrado seu efeito benéfico nos resultados do terceiro ensaio. A menor instabilidade também foi observada com o tamponamento decorrente da adição de $0,2 \mathrm{~mL}$ de ácido acético glacial e acetato de sódio no mesmo ensaio. As adições de sulfato de magnésio e citrato de sódio promoveram uma eficiente partição líquido-líquido, facilitando a remoção de componentes polares da matriz. ${ }^{34}$ No entanto, não foi observada diferença significativa quando da adição do citrato de sódio.

A centrifugação forneceu uma separação física das fases orgânica e aquosa, além de simultaneamente separar a fase sólida dispersiva. Estas etapas são empregadas por autores que determinaram pesticidas $^{24}$ e a centrifugação foi também determinante para a eficiência da extração neste caso. Extratos mais limpos foram verificados após a adição de terra diatomácea, ${ }^{35}$ que atua limitando a interferência de açúcares e lipídios. ${ }^{36}$

O método QuEChERS adequado neste estudo para extração simultânea de ocratoxina A e citrinina consistiu nas seguintes etapas: 
Tabela 3. Variáveis e recuperação da ocratoxina A para o planejamento fatorial $2^{4}$ completo

\begin{tabular}{|c|c|c|c|c|c|}
\hline Experimentos & Adição de água (mL) & Ácido acético $(\mathrm{mL})$ & $\begin{array}{c}\text { Sulfato, acetato e citrato } \\
(\mathrm{g})\end{array}$ & Terra diatomácea (mg) & Recuperação (\%) \\
\hline 1 & $-1(10)$ & $-1(0)$ & $-1(3+1,7+1)$ & $-1(200)$ & 42 \\
\hline 2 & $-1(10)$ & $-1(0)$ & $-1(3+1,7+1)$ & $+1(500)$ & 70 \\
\hline 3 & $-1(10)$ & $-1(0)$ & $+1(6+3,4+2)$ & $-1(200)$ & 59 \\
\hline 4 & $-1(10)$ & $-1(0)$ & $+1(6+3,4+2)$ & $+1(500)$ & 82 \\
\hline 5 & $-1(10)$ & $+1(0,1)$ & $-1(3+1,7+1)$ & $-1(200)$ & 105 \\
\hline 6 & $-1(10)$ & $+1(0,1)$ & $-1(3+1,7+1)$ & $+1(500)$ & 16 \\
\hline 7 & $-1(10)$ & $+1(0,1)$ & $+1(6+3,4+2)$ & $-1(200)$ & 73 \\
\hline 8 & $-1(10)$ & $+1(0,1)$ & $+1(6+3,4+2)$ & $+1(500)$ & 38 \\
\hline 9 & $+1(20)$ & $-1(0)$ & $-1(3+1,7+1)$ & $-1(200)$ & 83 \\
\hline 10 & $+1(20)$ & $-1(0)$ & $-1(3+1,7+1)$ & $+1(500)$ & 92 \\
\hline 11 & $+1(20)$ & $-1(0)$ & $+1(6+3,4+2)$ & $-1(200)$ & 72 \\
\hline 12 & $+1(20)$ & $-1(0)$ & $+1(6+3,4+2)$ & $+1(500)$ & 91 \\
\hline 13 & $+1(20)$ & $+1(0,1)$ & $-1(3+1,7+1)$ & $-1(200)$ & 105 \\
\hline 14 & $+1(20)$ & $+1(0,1)$ & $-1(3+1,7+1)$ & $+1(500)$ & 100 \\
\hline 15 & $+1(20)$ & $+1(0,1)$ & $+1(6+3,4+2)$ & $-1(200)$ & 88 \\
\hline 16 & $+1(20)$ & $+1(0,1)$ & $+1(6+3,4+2)$ & $+1(500)$ & 51 \\
\hline 17 & $0(15)$ & $0(0,05)$ & $0(4,5+2,6+1,5)$ & $0(200)$ & 60 \\
\hline 18 & $0(15)$ & $0(0,05)$ & $0(4,5+2,6+1,5)$ & $0(200)$ & 69 \\
\hline 19 & $0(15)$ & $0(0,05)$ & $0(4,5+2,6+1,5)$ & $0(200)$ & 58 \\
\hline 20 & $0(15)$ & $0(0,05)$ & $0(4,5+2,6+1,5)$ & $0(500)$ & 86 \\
\hline 21 & $0(15)$ & $0(0,05)$ & $0(4,5+2,6+1,5)$ & $0(500)$ & 78 \\
\hline 22 & $0(15)$ & $0(0,05)$ & $0(4,5+2,6+1,5)$ & $0(500)$ & 84 \\
\hline
\end{tabular}

-1 e +1: Níveis estudados e 0: pontos centrais.

Tabela 4. Dados de ANOVA para planejamento experimental avaliando as variáveis significativas no intervalo de confiança de $90 \%$

\begin{tabular}{lcccccc}
\hline Fonte de variação & Soma quadrática & Grau de liberdade & Média quadrática & Teste $F$ & $F_{\text {tab }(95 \%)}$ & Fcal/Ftab \\
\hline Regressão & 7454,3 & 4 & 1863,5 & 9,7 & & \\
Resíduo & 3263,0 & 17 & 191,9 & & \\
Falta de ajuste & 2539,5 & & & & \\
Erro puro & 723,5 & & & & \\
Total & 10717,3 & 21 & & & \\
\hline
\end{tabular}

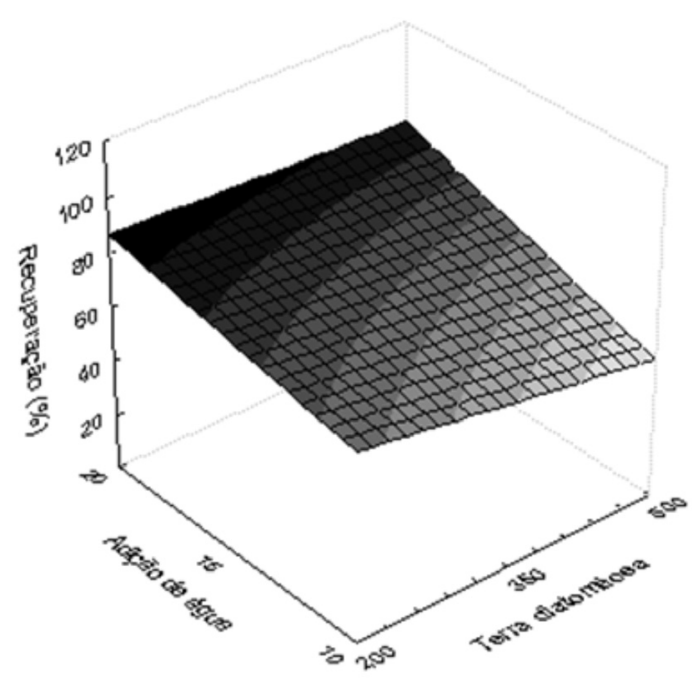

Figura 1. Superfície de resposta obtida para a recuperação no intervalo de confiança de $90 \%$

foram tomadas $10,00 \mathrm{~g}$ da fração de amostra de arroz triturado em moinho de facas e proveniente da fração granulométrica $0,5 \mathrm{~mm}$, colocadas em um erlenmeyer ao qual foram adicionados $20 \mathrm{~mL}$ de água destilada e $20 \mathrm{~mL}$ de acetonitrila acidificada com $0,2 \mathrm{~mL}$ de ácido acético glacial. A homogeneização da mistura foi realizada em mesa agitadora orbital durante $10 \mathrm{~min}$, a $200 \mathrm{rpm}$, com adição de $1,50 \mathrm{~g}$ de sulfato de magnésio e $0,85 \mathrm{~g}$ de acetato de sódio e nova agitação durante $10 \mathrm{~min}$, a $200 \mathrm{rpm}$. O material foi centrifugado por $8 \mathrm{~min}$, a $3220^{\prime} \mathrm{g}$, ao sobrenadante foram adicionados $0,30 \mathrm{~g}$ de sulfato de magnésio e $0,20 \mathrm{~g}$ de terra diatomácea, agitados manualmente durante 1 min e nova centrifugação por 8 min a $3220^{\prime} \mathrm{g}$. O sobrenadante foi seco em concentrador sob nitrogênio. Todas as extrações foram realizadas em triplicata. Na Figura 2 são apresentadas as condições estabelecidas para o método QuEChERS neste estudo em comparação com o método original. ${ }^{24}$

Alguns autores avaliaram a modificação do método QuEChERS para extração de micotoxinas de Fusarium em amostras de trigo. A otimização foi alcançada com a utilização de diferentes solventes, chegando-se a uma recuperação de $86-108 \%$ para uma mistura de metanol/acetonitrila 85/15 (v/v). ${ }^{37}$

As condições cromatográficas, conforme esperado, permitiram a recuperação acima de $70 \%$ para as duas micotoxinas, com limites de quantificação inferiores aos estabelecidos em legislações distintas.

Nestas condições, o método QuEChERS foi aplicado a 38 amostras que já haviam sido determinadas por CLAE-DAD com $5 \%$ de amostras contaminadas. ${ }^{38}$ Associando QuEChERS ao CLAE-IENEM/EM foi possível detectar que das 38 amostras $16 \%$ apresentaram contaminação com ocratoxina A, sendo que 3 estavam com níveis abaixo do limite estabelecido pela União Europeia $(5 \mu \mathrm{g} / \mathrm{kg})$. Em 


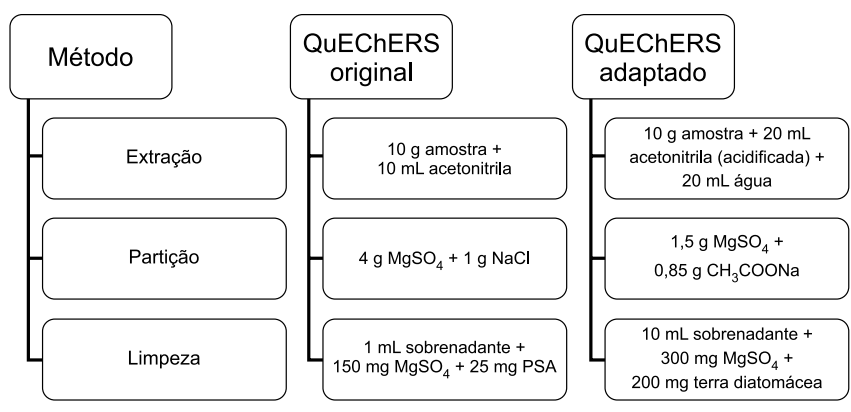

Figura 2. Representação das etapas do método QuEChERS original e adaptado neste estudo

uma única amostra o nível alcançou $560 \mu \mathrm{g} / \mathrm{kg}$, estando 112 vezes acima do LMT estabelecido. ${ }^{30}$ Duas outras amostras apresentaram contaminação por citrinina, nos níveis de 12 e $129 \mu \mathrm{g} / \mathrm{kg}$ a contaminação em co-ocorrência das duas micotoxinas não foi verificada em nenhuma das amostras analisadas; nenhuma amostra apresentou contaminação simultânea com ambas as micotoxinas.

Foram estudados três métodos para extração simultânea de 136 pesticidas, 36 toxinas naturais e 86 medicamentos veterinários em alimentos para consumo animal e mel. Os três procedimentos, incluindo o método QuEChERS, apresentaram recuperações que variaram de 70 a $120 \%$ para todos os analitos. ${ }^{39} \mathrm{O}$ método QuEChERS e o procedimento de extração acelerada por solvente (ASE - Accelerated Solvent Extraction) foram estudados para extração de 17 micotoxinas, incluindo ocratoxina $\mathrm{A}$, em alimentos à base de cereais. Ambos tiveram desempenhos semelhantes em relação à linearidade, precisão e limite de quantificação, no entanto o método QuEChERS mostrou-se mais fácil, com uma recuperação que variou de 73-130\% e ainda teve um maior rendimento de amostra se comparado com ASE. ${ }^{40}$

Apesar da ampla ênfase que vem sendo dada ao emprego do método QuEChERS, poucos pesquisadores empregaram planejamento experimental para otimizar a combinação de variáveis do método.

\section{CONCLUSÃO}

O planejamento experimental realizado mostrou-se uma ferramenta importante para otimizar e construir o modelo preditivo dentro da faixa ótima para a extração das duas micotoxinas simultaneamente. As condições otimizadas ocorreram quando foi utilizada a maior quantidade de água $(20 \mathrm{~mL})$ e menor quantidade de terra diatomácea (200 mg). As recuperações de citrinina e ocratoxina A foram de 78$105 \%$. Os resultados encontrados mostram a importância do avanço da pesquisa na avaliação da ocorrência e co-ocorrência simultânea de ocratoxina A e citrinina em amostras de arroz, pois, estas estavam presentes em aproximadamente $16 \%$ das amostras.

\section{AGRADECIMENTOS}

Ao Conselho Nacional de Desenvolvimento Científico e Tecnológico pelo apoio financeiro e ao Instituto Rio Grandense do Arroz pelas amostras.

\section{REFERÊNCIAS}

1. Bräse, S.; Encinas, A.; Keck, J.; Nising, C. F.; Chem. Rev. 2009, 109, 3903.

2. Clark, H. A.; Snedeker, S. M.; J. Toxicol. Environ. Health, Part B 2006, 9, 265 .

3. Richard, J. J.; Int. J. Food Microbiol. 2007, 119, 3.

4. Paterson, R. P. M.; Lima, N.; J. Appl. Microbiol. 2009, 106, 1070.
5. Martins, M. L.; Gimeno, H. M.; Martins, H. M.; Bernardo, F.; Food Addit. Contam. 2002, 19, 568.

6. Speijers, G. J. A; Speijeirs, M. H. M.; Toxicol. Lett. 2004, 153, 91.

7. Nielsen, K. F.; Smedsgaard, J.; J. Chromatogr. 2003, 1002, 111.

8. Li, S.; Marquardt, R. R.; Frohlich, A. A.; Food. Chem. Toxicol. 2000, 38, 141.

9. Amezqueta, S.; Gonzalez-Penas, E.; Murillo-Arbizu, E.; De Cerain, A. L.; Food Control 2009, 20, 326.

10. FAO - Food and Agriculture Organization; Food and Nutrition Paper, (2001), N 64, 357 Roma, 1997.

11. Curtui, V. G.; Gareis, M.; Food Addit. Contam. 2001, 18, 635.

12. Almela, L.; Robe, V.; Sanchez, B.; Torella, F.; Perez-Lopez, J.; Gabaldon, J. A.; Food Microbiol. 2007, 24, 319.

13. Ribeiro, J. M.; Cavaglieri, L. R.; Fraga, M. E.; Direito, G. M.; Dalcero, A. M.; Rosa, C. A.; Lett. Appl. Microbiol. 2006, 42, 179.

14. Xu, B.; Jia, X.; Gu, L.; Sung, C.; Food Control 2006, 17, 271.

15. Shu, P. Y.; Lin, C. H.; Anal. Sci. 2002, 18, 283.

16. Meister, U.; Eur. Food Res. Technol. 2004, 218, 394.

17. Aziz, N. H.; Moussa, L. A. A.; Food Control 2002, 13, 281.

18. Bailly, J. D.; Querin, A.; Le Bars-Bailly, S.; Benard, G.; Guerre, P.; J. Food Prot. 2002, 65, 1317.

19. Ren, Y.; Zhang, Y.; Shao, S.; Cai, Z.; Feng, L.; Pan, H.; Wang, Z.; J. Chromatogr., A 2007, 1143, 48.

20. Gobel, R.; Lusky, K.; J. AOAC Int. 2004, 87, 411.

21. Saint-Marcoux, F.; Lachatre, G.; J. Am. Soc. Mass Spectrom. 2003, 14, 14.

22. Rahmani, A.; Jinap, S.; Soleimany, F.; Compr. Rev. Food Sci. F. 2009, 8 , 202.

23. Sulyok, M.; Krska, R.; Schuhmacher, R.; Food Chem. 2010, 119, 408.

24. Anastassiades, M.; Lehotay, S. J.; Stajnbaher, D.; Schenck, F. J.; J. AOAC Int. 2003, 86, 412.

25. Prestes, O. P.; Friggi, C. A.; Adaime, M. B.; Zanella, R.; Quim. Nova 2009, 32, 1620

26. AOAC - Association Official Analytical Chemists; Official methods of analysis of AOAC international, $17^{\text {th }}$ ed., AOAC: Arlington, 2000.

27. INMETRO - Instituto Nacional de Metrologia, Normalização e Qualidade Industrial; Orientações sobre Validação de Métodos de Ensaios Químicos, DOQ-CGCRE-008, Revisão 01, 2003.

28. ANVISA - Agência Nacional de Vigilância Sanitária; Guia para Validação de Métodos Analíticos e Bioanalíticos, RE n 889, de 29/05/2003.

29. Ribani, M.; Bottoli, C. B. G.; Collins, C. H.; Jardim, I. C. S. F.; Melo, L. F. C.; Quim. Nova 2004, 27, 771.

30. ANVISA - Agência Nacional de Vigilância Sanitária; $R E n^{\circ} 7$, de 02/02/2011.

31. Zachariasova, M.; Lacina, O.; Malachova, A.; Kostelanska, M.; Poustka, J.; Godula, M.; Hajslova, J.; Anal. Chim. Acta 2010, 662, 51.

32. Cunha, F.; Fernandes, J. O.; J. Sep. Sci. 2010, 33, 600.

33. EU (Commission regulation (EC) $\mathrm{N}^{\circ} 1881 / 2006$, de 19/12/2006, que fixa os teores máximos de determinadas quantidades de gêneros alimentícios), Jornal Oficial da União Europeia, publicado em 20/12/2006.

34. Lehotay, S. J.; Mastovska, K.; Lightfield, A. R.; J. AOAC Int. 2005, 88, 615.

35. Caldas, E. D.; Silva, S. C.; Oliveira, J. N.; Revista Saúde Pública 2002 , 36,319 .

36. Badiale-Furlong, E.; Soares, L. M. V.; J. AOAC Int. 1995, 78, 386.

37. Sospedra, I.; Blesa, J.; Soriano, J. M.; Mañes, J.; J. Chromatogr., A 2010, 1217,1437

38. Hackbart, H. C. S.; Prietto, L.; Primel, E. G.; Garda-Buffon, J.; Badiale-Furlong, E.; J. Braz. Chem. Soc. 2012, 23, 103.

39. Mol, H. G. J.; Plaza-Bolaños, P.; Zomer, P.; Rijk, T. C.; Stolker, A. A. M.; Mulder, P. P. J.; Anal. Chem. 2008, 80, 9450.

40. Desmarchelier, A.; Oberson, J.; Tella, P.; Gremaud, E.; Seefelder, W.; Mottier P.; J. Agric. Food Chem. 2010, 58, 7510. 